\title{
The degree of natriuretic peptide system activation in stable and high risk CHF characterized by increased cystatin $C$ is not reflected in CGMP activation
}

\author{
John A Schirger ${ }^{1}$, Horng H Chen ${ }^{1}$, Josh Slusser ${ }^{2}$, David Hodge ${ }^{2}$, \\ Denise Heublein ${ }^{1}$, Alessandro Cataliotti ${ }^{1}$, Sharon Sandberg ${ }^{1}$ and \\ John C Burnett Jr*1
}

\author{
Address: ${ }^{1}$ Cardiovascular Diseases, Cardiorenal Research Laboratory, Mayo Clinic, Rochester, MN 55905, USA and ${ }^{2}$ Department of Biostatistics, \\ Mayo Clinic, Rochester, MN 55905, USA \\ Email: John C Burnett* - schirger.john@mayo.edu \\ * Corresponding author
}

\author{
from 4th International Conference of cGMP Generators, Effectors and Therapeutic Implications \\ Regensburg, Germany. 19-21 June 2009 \\ Published: II August 2009 \\ BMC Pharmacology 2009, 9(Suppl I):P63 doi:10.1186/I47I-2210-9-SI-P63
}

This abstract is available from: http://www.biomedcentral.com/I47I-22I0/9/SI/P63

(c) 2009 Schirger et al; licensee BioMed Central Ltd.

\begin{abstract}
Background
Cardiorenal syndrome (CRS) is characterized by neurohumoral activation, sodium retention, ventricular dysfunction and congestion, and renal insufficiency (RI). The congestive heart failure (CHF) component evolves from asymptomatic, to stable symptomatic to overt symptomatic CHF. The natriuretic peptide system (NPS), including atrial (ANP) and brain (BNP) natriuretic peptides is progressively activated during progression of CHF. Less is known about the second messenger, cGMP, of the NPS across the spectrum of CHF. RI in CHF remains an important clinical challenge and Cystatin $\mathrm{C}$ is emerging as an important marker of RI. Further work is needed in characterizing Cystatin C across the spectrum of CHF and CRS.
\end{abstract}

\section{Methods}

We prospectively looked at the NPS, including ANP, NTproANP, BNP, and NT-proBNP, as well as plasma cGMP, NPS to cGMP ratios and Cystatin $\mathrm{C}$ in three groups of subjects: normal volunteers $(\mathrm{n}=10)$, stable CHF patients ( $\mathrm{n}$ $=19$ ), and higher risk CHF patients (based on recent hospitalization for $\mathrm{CHF}, \mathrm{n}=20$ ). The groups are reported in that order below. Statistical analysis was carried out by ANOVA with $\mathrm{p}<0.05$ accepted as significant $\left({ }^{*}\right)$.

\section{Results}

Each component of the NPS increased in the plasma across the three groups with highest levels in the high risk CHF. However, CGMP levels did not vary significantly across groups ( $626 \pm 303$ vs $914 \pm 521$ vs $959 \pm 509 \mathrm{pmol} /$ $\mathrm{mL}, \mathrm{NS})$. The ratio NT-proANP/cGMP increased across the 3 groups $\left(0.60 \pm 0.55\right.$ vs $1.02 \pm 0.52$ vs $\left.1.46 \pm 1.08,{ }^{*}\right)$, but the ratios of ANP/cGMP, BNP/cGMP, and NT-proBNP/ cGMP did not vary significantly across groups. Importantly, the mean Cystatin C levels increased with stable and high risk CHF groups $(0.9 \pm 0.1$ vs $1.4 \pm 0.3$ vs $1.8 \pm$ $\left.0.7,{ }^{*}\right)$.

\section{Conclusion}

Despite the increase of each component of the NPS measured in the stable and high risk CHF, cGMP did not increase significantly across the same groups. Except for the ratio NT-proANP/cGMP, the ratios of the NPS to cGMP did not vary across groups either, suggesting the need to further refine our understanding of the coupling of the NPS with its second messenger, cGMP. Importantly, we report for the first time that Cystatin $\mathrm{C}$ varies across these phenotypes of $\mathrm{CHF}$, suggesting that it may be an important marker of the development of CRS. 